\title{
ATENDimento PSICOLÓGICO A PACIENTES COM INSUFICIÊNCIA RENAL CRÔNICA: EM BUSCA DE AJUSTAMENTO PSICOLÓGICO
}

\author{
Marineia Crosara de Resende* \\ Francisco Assis dos Santos** \\ Melissa Macedo de Souza*** \\ Thatianna Pereira Marques****
}

\section{Resumo}

As doenças crônicas, entre elas a insuficiência renal crônica, têm recebido grande atenção dos profissionais de saúde nas últimas décadas devido ao importante papel desempenhado na morbimortalidade da população mundial. Este trabalho pretende apresentar um relato de experiência sobre o atendimento psicológico, realizado numa clínica de hemodiálise, de 26 pacientes com idades entre 15 e 80 anos, a maioria mulheres (69,2\%), casados $(53,8 \%)$, que não trabalham $(92,3 \%)$ e aposentados $(57,7 \%)$, fazendo hemodiálise três vezes por semana. No lugar de reagir passivamente a eventos negativos, indivíduos podem se comportar de modos que aumentem seu bem-estar em tempos de desafios e mudanças, como no caso de se ter uma insuficiência renal crônica. A forma como as pessoas lidam com o sofrimento e seus benefícios pode trazer possibilidades de ajustamento e crescimento pessoal frente à adversidade.

Palavras-chave: psicologia da saúde, ajustamento psicológico, atendimento psicológico, insuficiência renal crônica, hemodiálise

* Psicóloga; Professora de Psicologia do Centro Universitário do Triângulo; Mestre em Gerontologia e Doutora em Educação pela Universidade Estadual de Campinas (UNICAMP).

** Psicólogo do Centro Universitário do Triângulo.

*** Psicóloga do Centro Universitário do Triângulo.

**** Psicóloga do Centro Universitário do Triângulo. 


\begin{abstract}
PSYCHOLOGICAL TREATMENT FOR PATIENTS WITH CHRONIC KIDNEY DISEASE: SEARCHING FOR PSYCHOLOGICAL ADJUSTMENT

Chronic illnesses, such as kidney disease, have received great attention from health professionals in the last decades. This work intends to present an experience with psychological care, in a hemodialysis clinic, with 26 patients aged between 15 and 80 years, the majority of which were women (69,2\%), married (53,8\%), did not work (92,3\%), or were retired (57,7\%), taking hemodialysis three times a week. Instead of reacting negatively to life events, individuals can behave in ways that improve their well-being in times of transformation and change, as is the case when one has a chronic kidney disease. The manner in which the individual deals with suffering and its benefits can bring possibilities of psychological adjustment and personal growth in face of adversity.
\end{abstract}

Keywords: health psychology, psychological adjustment, psychological care, chronic kidney disease, hemodialysis

As doenças crônicas têm recebido grande atenção dos profissionais de saúde nas últimas décadas pelo fato do importante papel desempenhado na morbimortalidade da população mundial. Entre essas doenças está a insuficiência renal crônica, considerada uma condição sem alternativas de melhoras rápidas, de evolução progressiva, que causa problemas médicos, sociais e econômicos (Martins \& Cesarino, 2005).

A doença renal é considerada um grande problema de saúde pública, porque causa elevadas taxas de morbidade e mortalidade e, além disso, tem impacto negativo sobre a qualidade de vida relacionada à saúde, que é a percepção da pessoa de sua saúde por meio de uma avaliação subjetiva de seus sintomas, satisfação e adesão ao tratamento (Martins \& Cesarino, 2005). Segundo a Sociedade Brasileira de Nefrologia, no mundo, cerca de 1,2 milhão de pessoas encontram-se sob tratamento dialítico. No Brasil, são aproximadamente 54,5 mil pessoas, destas, 48.875 em hemodiálise e 5.649 em diálise peritoneal. O número de pacientes em programa dialítico cresce no Brasil à média de $10 \%$, às custas de uma incidência de mais de cem pacientes novos por milhão de habitantes/ano (Vieira, Gomes, Frota, Andrade, Vieira, Moura \& Vieira, 2005).

A insuficiência renal crônica é o resultado final do comprometimento da função renal por diversas doenças que acometem os rins, de maneira rápida ou lenta e progressiva, que tornam o rim incapaz de realizar as suas funções. O ritmo de tal progressão depende da doença original e de causas agravantes, como hipertensão, infecção urinária, nefrite, gota e diabetes (Kusumota, Rodrigues \& Marques, 2004). Geralmente, quando surge uma doença renal, ela ocorre nos dois 
rins, raramente atingindo um só. Quando o rim adoece por uma causa crônica e progressiva, a perda da função renal pode ser lenta e prolongada (Martins \& Cesarino, 2005).

A adesão ao tratamento da doença crônica significa aceitar a terapêutica proposta e segui-la adequadamente. Vários fatores influenciam a adesão, tais como a característica da terapia, as peculiaridades do paciente, aspectos do relacionamento com a equipe multidisciplinar, variáveis socioeconômicas, entre outras (Rapley, 1997, citado por Kurita \& Pimenta, 2003).

Para Cesarino e Casagrande (1998), a Insuficiência Renal Crônica e o tratamento hemodialítico provocam uma sucessão de situaçôes, para o paciente renal crônico, que compromete, além do aspecto físico, o psicológico, com repercussões pessoais, familiares e sociais. Por isso, além do acompanhamento médico às doenças renais para prolongar o bom funcionamento do rim, mesmo com certos graus de insuficiência (Martins \& Cesarino, 2005), é de fundamental importância o atendimento psicológico.

$\mathrm{O}$ atendimento e tratamento dos aspectos psicológicos em torno do adoecimento - que se dá quando o sujeito humano, carregado de subjetividade, esbarra em um real, de natureza patológica, denominado doença, presente em seu próprio corpo - produz uma infinidade de aspectos psicológicos que podem se evidenciar no paciente, na família ou na equipe de profissionais (Simonetti, 2004).

A pessoa com insuficiência renal crônica vivencia uma brusca mudança no seu viver, convive com limitações, com um pensar na morte, com o tratamento doloroso que é a hemodiálise (Cesarino \& Casagrande, 1998). A hemodiálise consiste na diálise promovida por uma máquina, que filtra o sangue fora do organismo, é realizada em média três vezes por semana, num período de três a cinco horas por sessão, dependendo das necessidades individuais (Kusumota, Rodrigues \& Marques, 2004). Sendo assim, o tratamento hemodialítico é responsável por um cotidiano monótono e restrito, e as atividades desses indivíduos são limitadas após o início do mesmo, favorecendo o sedentarismo e a deficiência funcional, fatores que se refletem na vida diária do paciente (Martins \& Cesarino, 2005).

O desenvolvimento de doenças crônicas e incapacidades na vida adulta são associados à deterioração, à redução de competências, ao aumento da necessidade de ajuda, à dor física e emocional resultante em perda da independência e ao aumento de necessidade de assistência (Gignac \& Cott, 1998).

Lima (1989, citado por Cesarino \& Casagrande, 1998) afirma que os pacientes com insuficiência renal crônica acabam tornando-se pessoas desanimadas, desesperadas e, muitas vezes, por estas razões ou por falta de orientação, acabam abandonando o tratamento ou não dando importância aos cuidados constantes 
que deveriam ter. É necessário estimular as capacidades a se adaptarem de maneira positiva ao novo estilo de vida, assumindo o controle de seu tratamento, de sua vida e, conseqüentemente, melhorando seu ajustamento (Resende, 2006).

$\mathrm{O}$ ajustamento pode ser biologicamente definido como uma resposta a uma mudança no ambiente que permite ao organismo tornar-se mais adaptado àquela mudança. Esta definição tem uma dimensão temporal, uma vez que considera que o ajustamento acontece com o passar do tempo. Em psicologia, o conceito de ajustamento refere-se basicamente a um estado desejável (Brennan, 2001, citado por Sharpe \& Curran, 2006).

Ryff (1989) propôs um modelo multidimensional de bem-estar psicológico como sinônimo de ajustamento, uma condição do self relacionada a seis domínios do funcionamento psicológico: ter uma atitude positiva perante si próprio e sua vida passada (auto-aceitação); ter metas e objetivos que confiram significado à vida (propósito na vida); estar apto a lidar com as exigências complexas da vida diária (domínio sobre o ambiente); ter um sentido de desenvolvimento contínuo e auto-realização (crescimento pessoal); possuir vínculos de afeto e confiança com os outros (relaçôes positivas com os outros); e estar apto a seguir suas próprias convicções (autonomia).

A auto-aceitação significa reconhecer e aceitar características positivas e negativas. O sentimento de aceitação gera estima, confiança e segurança em si e nos outros (Ryff, 1989). A forma como o indivíduo se vê e se descreve, suas crenças sobre como é visto pelos outros, a similaridade que percebe entre o que é e o que acredita que os outros pensam dele e o grau em que valoriza suas competências em comparação com os outros são forças orientadoras para os esforços de adaptação (Leão Júnior \& Resende, 2004).

É necessário que a pessoa com alguma incapacidade aceite sua condição, como relata Frankl (1990), já que mesmo diante de adversidades e de sofrimento é possível encontrar sentido. O autor afirma que é importante encontrar sentido no sofrimento para que a pessoa consiga superá-lo da melhor forma possível. Tirar lições positivas das experiências dolorosas faz a pessoa crescer na dimensão mais profunda que um ser humano pode alcançar: dar sentido à sua dor, por pior que ela possa parecer. "Sempre e em toda parte, a pessoa está colocada diante da decisão de transformar a sua situação de mero sofrimento numa realização interior de valores" (Frankl, 1999: 68).

Janoff-Bulman e Frantz (1997, citados por Pakenham, 2005) propuseram que a adaptação positiva em face da adversidade envolve uma tentativa de encontrar sentido num evento traumático e achar algum benefício nesta experiência. A pessoa percebe que há sentido em sua vida presente e passada, tem crenças que 
dão propósito à vida, acredita que a vida é significativa e, com todos os obstáculos, ainda encontra motivos para continuar vivendo (Ryff, 1989).

O sentido de vida habilita a pessoa a manter sua saúde mental e sua integridade ainda que em condições adversas, como no caso de pessoas que viveram em campos de concentração. A busca e a realização do sentido pessoal representam uma nova perspectiva de promoção de bem-estar, de enfrentamento e de adaptação, principalmente para as pessoas em condição de fragilidade, uma das poucas áreas em que o indivíduo experimenta uma continuidade no crescimento pessoal, mesmo vivendo as perdas e o declínio físico (Freire \& Resende, 2001).

Borges e Martins (2001) realizaram uma pesquisa com pacientes renais crônicos atendidos em uma clínica de hemodiálise de São Paulo através de entrevistas semidirigidas com 5 pessoas. Os resultados indicaram que as pessoas relatam sentir várias perdas: a) ocasionadas indiretamente pela doença, tais como perdas físicas, e as interacionais, ou seja, perda da visão, da capacidade de realizar algumas atividades, afastamento de amigos, etc; b) perdas que não estão diretamente relacionadas ao tratamento dialítico, entretanto são consideradas significativas para o paciente, já que a maioria delas é definitiva, tais como o fato de ter que comparecer às sessôes de diálises semanais, de tomar uma série de medicamentos, seguir dieta rígida, restrições de atividades e conseqüentes mudanças de hábitos. Por isso a necessidade de aprender a lidar com aspectos do ambiente.

Segundo Ryff (1989), domínio sobre o ambiente significa ter competência para manejar o ambiente, usufruir as oportunidades que surgem ao seu redor, apresentar habilidades para escolher ou criar contextos adequados às suas necessidades e valores, eliminar barreiras e fazer adaptações pessoais ou no ambiente, quando necessárias. É preciso que o ambiente seja compatível com as capacidades físicas e com as competências comportamentais das pessoas.

As necessidades presentes, o ambiente e os agentes desencadeadores de estresse dependem de como os indivíduos atendem às necessidades de auto-atualização. Esta é possível quando existe um equilíbrio entre as necessidades individuais, os fatores geradores de estresse e a capacidade de adaptação às alterações do corpo e do ambiente (Perry \& Potter, 1999, citados por Martins, Cunha \& Coelho, 2005), particularmente quando se conta com uma rede de relaçóes positivas.

As relações positivas com os outros engloba ter com estes uma relação de qualidade, calorosa, satisfatória e verdadeira; preocupar-se com o bem-estar do outro; ser capaz de manter relações afetuosas e agradáveis, sejam elas familiares, de intimidade ou de amizade (Ryff, 1989).

Zimmermann, Carvalho e Mari (2004) citam Kimmel (2000), que relata que o suporte social está vinculado ao aumento da sobrevida em pacientes com 
insuficiência renal crônica em hemodiálise. Citam também a pesquisa de Shidler e colaboradores (1998), que, estudando 50 pacientes com insuficiência renal crônica, afirmam que o suporte social não apresenta uma ação protetora maior para situações de estresse leves, mas previne ou reduz os efeitos negativos em situações de estresse elevado.

Segundo Abrunheiro (2005), o suporte social tem sido um dos principais conceitos da Psicologia da saúde, pois em situação de estresse, ou de crise, alivia e até mesmo inibe o desenvolvimento de doenças; além disso, quando o indivíduo já se encontra doente, tem um papel positivo em sua recuperação.

Andrade e Vaitsman (2002) afirmam que, segundo algumas pesquisas, a pobreza de relações sociais constitui fator de risco à saúde comparável a outros que são comprovadamente nocivos. Isso porque a condição de enfermidade, por si só, coloca os indivíduos diante de limitações, impedimentos e situações que mudam a relação da pessoa com o trabalho, com seus familiares, com amigos e parceiros, bem como abalam sua identidade. Muitas vezes, o paciente experimenta fragilização da identidade, do próprio sentido da vida e da capacidade de resolver problemas que o afetam pelo fato de tudo aquilo que organizava a sua identidade ter sido alterado de forma brusca com a presença da doença (Gibson, 1991, citado por Andrade \& Vaitsman, 2002).

Nesse sentido, o apoio social poderia ser um elemento a favorecer o processo no qual indivíduos, grupos sociais e organizaçōes passam a ganhar maior autonomia, mais controle sobre seus próprios destinos (Valla, 1999, citado por Andrade \& Vaitsman, 2002).

O fator autonomia também exerce uma influência importante na vida dos enfermos. A doença faz com que algumas pessoas deixem de trabalhar, sintam-se incapazes de participar de atividades sociais, deixem de lado atividades antes rotineiras, como encontros com amigos, idas a igreja, ou seja, restringem grandemente o acesso à interação social, provocando uma sensação de suporte reduzido que interfere de forma negativa em sua saúde (Abrunheiro, 2005).

A autonomia implica ser autodeterminado e independente, mesmo que para isso se necessite de apoio para operacionalizar escolhas e às vezes até para o exercício de atividades da vida diária; tomar decisóes de acordo com o que se acredita ser melhor, dentro de seus próprios padrōes; e não ser influenciado por pressões sociais para pensar e agir (Ryff, 1989).

Para Pakenham (2005), pacientes com largo histórico de problemas médicos relataram benefícios ou ganhos a partir de suas adversidades, inclusive percepções de crescimento pessoal, fortalecimento de relações e mudanças nas prioridades de vida e metas pessoais. 
No crescimento pessoal, a pessoa apresenta um senso de desenvolvimento e crescimento contínuo, está aberta a novas experiências, reconhece seu potencial de realização e suas mudanças refletem autoconhecimento e auto-eficácia (Ryff, 1989).

Bauer e McAdams (2004) citam Maslow (1968), que ressaltou que as pessoas são orientadas para o crescimento ou para a segurança em suas vidas e que uma orientação ao crescimento facilita o alcance de saúde psicológica e de bemestar. Psicólogos humanísticos reivindicam que as pessoas que pensam em suas vidas predominantemente em termos de certos valores (por exemplo, inteireza, justiça, autonomia, empatia) estão no caminho do crescimento pessoal para maiores níveis de saúde psicológica e bem-estar.

Cada uma das dimensões do bem-estar psicológico articula diferentes possibilidades de mudanças individuais, encontradas quando alguém procura funcionar de maneira positiva (Keyes, Shmotkin \& Ryff, 2002). Muito recentemente é que as atenções começaram a se voltar para uma terapêutica visando à qualidade de vida do paciente com insuficiência renal crônica como um fator relevante no cenário da terapêutica renal. Essa busca origina-se da constatação de que alcançar um estado de bem-estar físico e mental é possível, resultando na recuperação da autonomia, das atividades de trabalho e lazer, da preservação da esperança e do senso de utilidade destes indivíduos (Martins \& Cesarino, 2005).

Desta forma, tendo em vista a necessidade de pesquisas e intervenções no que tange à diversidade inerente à realidade de pessoas com insuficiência renal crônica, acredita-se ser de fundamental importância proporcionar a presença de um psicólogo na equipe de clínicas de hemodiálise. $\mathrm{O}$ atendimento psicológico auxilia a quebrar tabus e preconceitos, além de incentivar as pessoas a desenvolverem suas capacidades, levando-as a verem a doença sob outros ângulos. Em outras palavras, faz parte dos esforços que devem ser empreendidos na promoção de um atendimento integral, visando proporcionar mais qualidade de vida a estas pessoas.

A partir do exposto, pretende-se relatar a experiência de intervenção psicológica em uma clínica de hemodiálise enquanto atividade de Estágio profissionalizante do curso de Psicologia. Através dos atendimentos, pretendeu-se contribuir para um melhor ajustamento de cada pessoa à sua condição de ter que conviver por tempo indeterminado com a máquina, os sintomas advindos da hemodiálise e as mudanças de vida causadas pela insuficiência renal crônica; trabalhando aspectos singulares do adoecimento, ajudando-as a fortalecerem os mecanismos de auto-regulação do self e trabalhando suas atitudes em relação ao tratamento e à vida. 


\section{CaracterizaÇÃo dos ATENDIMENTOS}

O estágio profissionalizante na área de psicologia clínica-hospitalar é realizado por alunos dos últimos períodos do curso de Psicologia, com supervisão semanal de uma professora psicóloga com experiência em atendimentos a pessoas na condição de fragilidade, entre elas a insuficiência renal crônica, em uma clínica de hemodiálise na cidade de Uberlândia, Minas Gerais, por dois semestres. Esta clínica é particular, subsidiada pelo SUS (Sistema Único de Saúde), e conta com aproximadamente 126 pacientes, que são atendidos divididos em 6 turnos.

Este relato diz respeito aos atendimentos realizados por três estagiários, em três turnos, duas vezes por semana. O critério utilizado para avaliar a necessidade de atendimento psicológico foi a avaliação clínica realizada através de Entrevista clínica e a Escala de Bem-Estar Psicológico de Ryff (1989): com 18 itens, as respostas devem ser dadas numa escala Likert de cinco pontos ("pouquíssimo" a "muitíssimo"), avalia o bem-estar psicológico referente aos domínios auto-aceitação, autonomia, domínio sobre o ambiente, propósito na vida, crescimento pessoal e relação positiva com os outros.

\section{Os ATENDIMENTOS}

A partir dessa avaliação, que serviu também para o estabelecimento de vínculo com os pacientes, realizada num primeiro contato, foram selecionadas para atendimento 26 pessoas, com idade entre 15 e 80 anos, a maioria mulheres $(69,2 \%)$, casados $(53,8 \%)$, que não trabalhavam $(92,3 \%)$, aposentados $(57,7 \%)$, fazendo hemodiálise há mais de quatro anos $(46,0 \%)$ e em tratamento há menos de 1 ano $(11,0 \%)$. Durante o período de estágio, faleceram 2 pacientes que estavam em atendimento psicológico. Os atendimentos aconteciam uma ou duas vezes por semana, dependendo da queixa e da necessidade individual para acompanhamento. No decorrer das intervenções psicológicas, foram utilizadas também leitura de estórias, técnicas de relaxamento, e visualização.

$\mathrm{Na}$ avaliação através da Escala foi possível verificar que os participantes apresentaram bem-estar psicológico positivo (média de 3,88; $\mathrm{DP}=0,4$ ), dando indícios de ajustamento pessoal. Os resultados indicaram que os participantes vêem-se como estando em crescimento pessoal (média 3,5; $\mathrm{DP}=0,5$ ) e tendo um propósito na vida (média 3,5; DP=0,7). Apresentam boa auto-aceitação (média 3,3; $\mathrm{DP}=0,7$ ), domínio sobre o ambiente $(3,4 ; \mathrm{DP}=0,7)$ e autonomia (média 3,6; $\mathrm{DP}=0,7$ ).

Nos atendimentos focais, trabalhavam-se questôes que emergiam no momento do tratamento dialítico, ou seja, enquanto os pacientes permaneciam liga- 
dos à máquina fazendo hemodiálise, o que ocasionava algumas interrupções em função de procedimentos técnicos.

As principais queixas que apareceram nas entrevistas foram a necessidade de adaptação ao tratamento e à questão da morte, especificamente quando algum paciente vinha a óbito, o que gerava sentimentos de medo de ser "o próximo" e fantasias de angústia.

Pode-se perceber a dificuldade dos pacientes, bem como da equipe, em lidar com assuntos relacionados à morte, ficando uma sensação de que é um assunto "tabu". Quando algum paciente falecia, o movimento da equipe era de silêncio e agia-se como se nada tivesse acontecido; os sentimentos e as falas ficavam velados. Temas sobre a morte são constantes em pacientes com IRC, pois, apesar do progresso nos tratamentos renais, Martins e Cesarino (2005) enfatizam que a expectativa de vida de pacientes em estágio final de doença renal é muito menor do que a descrita nos diversos grupos da mesma faixa etária da população geral. Esse dado poderá desencadear, nos envolvidos com o tratamento, uma série de fantasias sobre a morte que precisam ser acompanhadas por um profissional da área psicológica.

O risco de morte nestes pacientes é particularmente elevado em diabéticos, em brancos e idosos. Tem sido também mostrado que o número de anos que o paciente permanece em tratamento dialítico está relacionado com o risco de morte. Portanto, o número de anos em diálise pode ajudar a estimar a probabilidade de um paciente com insuficiência renal permanecer vivo por um certo período de tempo, caso não seja possível oferecer-lhe um transplante renal (Lopes, Batista, Costa, Nery \& Lopes, 2003).

A morte faz parte da vida, assim como o sofrimento, e enfrentar a dor com dignidade é a tarefa mais difícil neste encontro. Às vezes a oportunidade de crescimento interior surge em situaçóes extremamente difíceis para a pessoa, como em momentos de doenças graves, tragédias sociais, ou eventos inesperados. Por exemplo, descobrir que se tem uma doença grave também pode ser um caminho para reflexões sobre os motivos ou objetivos para continuar vivendo (Freire, Resende \& Sommerhalder, 2000).

\section{CONSIDERAÇÕES FINAIS}

Para as pessoas com insuficiência renal crônica, o tratamento hemodialítico é inevitável e o transplante é casual (Machado \& Car, 2003). Assim, entre esta relação dialética, está a equipe de profissionais que precisa ampliar sua compreensão da árdua, triste, difícil e monótona realidade de lidar com a impotência frente à doença e às impossibilidades de transformação. 
$\mathrm{Na}$ convivência com estes pacientes, ficou clara a importância da intervenção psicológica em busca de solução das limitações provocadas pela IRC e pelo tratamento, sendo necessária a atuação constante de profissionais da Psicologia, sensíveis e dispostos a trabalhar em prol da melhor adesão do paciente ao tratamento dialítico, além de poderem contribuir para um maior entendimento e capacidade de enfrentamento, por parte dos pacientes, de sua atual condição.

Além disso, o profissional de Psicologia deve ser mais um profissional da equipe de saúde que atua, junto com a equipe multiprofissional, no relacionamento com os pacientes e nas dores emocionais que rondam o ambiente hospitalar. O psicólogo deverá atuar com o intuito de minimizar o impacto da doença na vida do paciente e da família, tentando impedir os efeitos iatrogênicos na busca de garantir a direção do tratamento junto com a equipe multidisciplinar.

O trabalho do Psicólogo na hemodiálise deve acontecer tanto na reestruturação psíquica do paciente, como também na manutenção do tratamento. A assistência psicológica junto aos pacientes renais crônicos poderá auxiliá-los a encarar sua condição numa outra perspectiva, ativando estratégias de enfrentamento que resgatem o bem-estar e promovam melhor qualidade de vida, descobrindo possibilidades na adversidade.

Há muito a ser feito no trabalho com pacientes em hemodiálise, como afirmam Neri e Fortes (2006). Ao vivenciar um evento negativo, tal como uma doença crônica que muitas vezes incapacita o paciente para certas atividades, o senso de controle do indivíduo tende a ser acionado, ou seja, seus recursos pessoais e sociais sofrem uma pressão para se adaptarem ao novo modelo. Para Silva e Varela (1999, citados por Rabelo \& Neri, 2005), a adaptação é a capacidade de maximizar as possibilidades individuais, reorganizando a vida frente às limitaçôes, ajustando-se às diversas situações individualmente ou com ajuda de outros. É nessa ajuda que o psicólogo tem sua grande importância, pois é sabido que muitas pessoas, diante de um problema com esse tipo de cronicidade (IRC), vivenciam uma brusca mudança no seu viver, limitações, pensamentos sobre morte, tratamento doloroso (Cesarino \& Casagrande, 1998), e não conseguem sozinhas encontrar forças para superá-la.

A maneira como uma pessoa assume o seu destino e com ele o sofrimento que lhe foi reservado é uma das muitas possibilidades de dar sentido à vida. A forma como experienciamos o sofrimento é pessoal e há distintas possibilidades para lidar com esta experiência, seja retirando do sofrimento lições que poderão levar ao crescimento pessoal e a repensar os valores principais da vida, seja se revoltando e caindo em desespero (Freire, Resende \& Sommerhalder, 2000). Frankl (1992) lembra que este desespero não tem apenas um significado trágico porque 
às vezes ele é necessário para que a pessoa resignifique sua vida. A crise pode ser o pilar mestre de uma mudança. Com ela a pessoa pode refletir sobre sua vida e mudar a forma de estar no mundo, ou, como coloca Xausa (1988: 164), "sofrer, pois, significa agir, crescer e amadurecer".

\section{REFERÊNCIAS BIBLIOGRÁFICAS}

Abrunheiro, L. M. M. (2005). A satisfação com o suporte social e a qualidade de vida no doente após transplante hepático. <www.psicologia.com.pt>. Acessado em 25/10/07.

Andrade, G. R. B. \& Vaitsman, J. (2002). Apoio social e redes: conectando solidariedade e saúde. Ciência e Saúde Coletiva, 7, 4, 925-934.

Bauer, J. J. \& McAdams, D. P. (2004). Growth goals, maturity, and well-being. Developmental Psychology, 40, 1, 114-127.

Borges, L. R. \& Martins, D. G. (2001). Clinica de hemodiálise: existe qualidade de vida? Boletim de Iniciação Cientifica em Psicologia, 2, 1, 42-58.

Cesarino, C. B. \& Casagrande, L. D. R. (1998). Paciente com insuficiência renal crônica em tratamento hemodialítico: atividade educativa do enfermeiro. Revista Latino-Americana de Enfermagem, 6, 4, 31-40.

Frankl, V. E. (1990). A questão do sentido em psicoterapia. Campinas: Papirus. . (1992). A presença ignorada de Deus. Petrópolis: Vozes.

- (1999). Em busca de sentido: um psicólogo no campo de concentração. Petrópolis: Vozes.

Freire, S. A. \& Resende, M. C. (2001). Sentido de vida e envelhecimento. Em Neri, A. L. (Org.). Maturidade e velhice (pp.71-98). Campinas: Papirus.

Freire, S. A.; Resende, M. C. \& Sommerhalder, C. (2000). Sentido de vida - indagações e perspectivas psicológicas. Cadernos de Psicologia da SBP, 1, 1, 11-18.

Gignac, M. A. M. \& Cott, C. A. (1998). Conceptual model of independence and dependence for adults with chronic physical illness and disability. Social Science \& Medicine, 41, 739-754.

Keyes, C. L. M.; Shmotkin, D. \& Ryff, C. D. (2002). Optimizing well-being: the empirical encounter of two traditions. Journal of Personality and Social Psychology, 82, 6, 10071022.

Kurita, G. P. \& Pimenta, C. A. M. (2003). Adesão ao tratamento da dor crônica: estudo de variáveis demográficas, terapêuticas e psicossociais. Arquivos de Neuropsiquiatria, 61, 2B, 416-425.

Kusumota L.; Rodrigues, R. A. P. \& Marques S. (2004). Idosos com insuficiência renal crônica: alteraçôes no estado de saúde. Revista Latino-Americana de Enfermagem, 12, 3, 525-32. 
Leão Júnior, R. \& Resende, M. C. (2004). Auto-eficácia e memória. Em Neri, A. L. \& Yassuda, M. S. (Orgs.). Velhice bem-sucedida: aspectos afetivos e cognitivos (pp. 227-240). Campinas: Papirus.

Lopes, A. A.; Batista, P. B. P.; Costa, F. A.; Nery, M. M. \& Lopes, G. B. (2003). Número de anos em tratamento dialítico crônico e risco de morte em pacientes com e sem diabetes melito. Revista da Associação Médica Brasileira, 49, 3, 266-269.

Machado, L. R. C. \& Car, M. R. (2003). A dialética da vida cotidiana de doentes com insuficiência renal crônica: entre o inevitável e o casual. Revista de Escola de Enfermagem USP, 37, 3, 27-35.

Martins, M. R. I. \& Cesarino, C. B. (2005). Qualidade de vida de pessoas com doença renal crônica em tratamento hemodialitico. Revista Latino-Americana de Enfermagem, $13,5,670-676$.

Martins, G.; Cunha, S. \& Coelho, R. (2005). A auto-actualização como motivação para aceitar a doença. Revista Portuguesa de Psicossomática, 17, 1-2, 115-123.

Neri, A. L. \& Fortes, A. C. G. (2006). A dinâmica estresse e enfrentamento na velhice e sua expressão no prestar cuidados a idosos no contexto da família. Em Freitas, E. V.; Py, L.; Cançado, F. A. X.; Doll, J. \& Gorzoni, M. L. (Eds.). Tratado de Geriatria e Gerontologia (pp. 1277-1288). Rio de Janeiro: Guanabara Koogan.

Pakenham, K. I. (2005). Benefit finding in multiple sclerosis and associations with positive and negative outcomes. Health Psychology, 24, 2, 123-132.

Rabelo, D. F. \& Neri, A. L. (2005). Recursos psicológicos e ajustamento pessoal frente a incapacidade funcional na velhice. Psicologia em Estudo (Maringá), 10, 3, 403-412.

Resende, M. C. (2006). Ajustamento psicológico, perspectiva de envelhecimento pessoal e satisfação com a vida em adultos e idosos com deficiência física. Tese de Doutorado. Curso de Pós-Graduação em Educação, Faculdade de Educação da UNICAMP, Campinas, SP.

Ryff, C. D. (1989). Happiness is everything, or is it? Explorations on the meaning of psychological well-being. Journal of Personality and Social Psychology, 57, 6, 1069-1081. Ryff, C. D. \& Keyes, C. L. M. (1995). The structure of psychological well-being revisited. Journal of Personality and Social Psychology, 69, 4, 719-727.

Sharpe, L. \& Curran, L. (2006). Understanding the process of adjustement to ilness. Social Science \& Medicine, 62, 1153-1166.

Simonetti, A. (2004). Manual de Psicologia Hospitalar: o mapa da doença. São Paulo: Casa do Psicólogo.

Vieira, W. P.; Gomes, K. W. P.; Frota, N. B.; Andrade, J. E. C. B.; Vieira, R. M. R. A.; Moura, F. E. A. \& Vieira, F. J. F. (2005). Manifestações musculoesqueléticas em pacientes submetidos à hemodiálise. Revista Brasileira de Reumatologia, 45, 6, 357-364.

Xausa, I. A. M. (1988). A psicologia do sentido da vida. Petrópolis: Vozes.

Psic. Clin., Rio de Janeiro, vol.19, N.2, P.87 - 99, 2007 
Zimmermann, P. R.; Carvalho, J. O. \& Mari, J. J. (2004). Impacto da depressão e outros fatores psicossociais no prognóstico de pacientes renais crônicos. Revista de Psiquiatria do Rio Grande do Sul, 26, 3, 312-318.

Recebido em 21 de abril de 2007 Aceito para publicação em 26 outubro de 2007 